\title{
An IoT-based Occupational Safety Management System in Cold Storage Facilities
}

\author{
Y.P. Tsang, K.L. Choy*, T.C. Poon, \\ G.T.S. Ho, C.H. Wu, H.Y. Lam \\ Department of Industrial and Systems \\ Engineering \\ The Hong Kong Polytechnic University \\ Hong Kong, China \\ *kl.choy@polyu.edu.hk
}

\author{
P.S. Koo \\ AOC Limited \\ Hong Kong, China
}

\author{
H.Y. Ho \\ Chevalier Cold Storage and Logistics \\ Limited \\ Hong Kong, China
}

\begin{abstract}
In the contemporary strategy of cold chain logistics, cold storage plays an important role to keep the inventory under the extreme environmental conditions. As the demand of cold storage services is growing rapidly nowadays, attention paid on occupational safety of warehouse workers is increasing under extreme working environment. Traditionally, the safety of workers are assessed by their experience and personal judgement. Without automatic data capturing tools, it is hard to monitor the actual health status of workers who may be dangerous when working too long in the cold storage facilities. In addition, there is a lack of prompt signal to managers and firstaid teams for instant treatment when the workers get cold injuries or illnesses. Therefore, the real-time health monitoring and positioning of the workers are in need. Nowadays, Internet of Things (IoT) is a mean of real-time interconnection system in which target objects are equipped with the identifying and sensing technologies. This paper presents an IoT-based occupational safety management system (IoT-OSMS) by using the identifying and sensing techniques to locate workers' positions and to guarantee the occupational safety. Bluetooth Low Energy (BLE), a kind of Radio Frequency Identification (RFID) solutions, is used to locate and collect the information of the workers inside the cold storage facilities. The workers' locations are calculated by using Trilateration with Taylor's series adjustment and Kalman filter. On the other hand, fuzzy logic technique is used to examine and generate the customized cold stress and monitoring review cycle. By integrating the positioning and Fuzzy logic approaches seamlessly, it is found that the occupational safety of warehouse workers can be guaranteed and monitored in the real-time situation.
\end{abstract}

Keywords-Occupational safety; Internet of Things; Bluetooth Low Energy; Fuzzy logic; Cold chain logistics.

\section{INTRODUCTION}

Due to the increasing concerns of food safety in Hong Kong, supply chain management has been developed to integrate with the refrigeration and environmental monitoring, especially in the storage and transportation. The storage and transportation play two important roles to maintain the good quality of the products and services [1]. Compared with the traditional supply chain management, products in cold supply chains usually have shorter shelf life as well as high sensitivity of environmental conditions, particularly in temperature and humidity [2]. The information flow in cold chain is highly concerned in aspects of product quality, costs incurred in the cold chain, and delivery and storage durations [3]. As the warehousing operations are relatively complicated involving receiving, put-away, sorting, picking and packing, the inventory will be placed in the cold storage environment for a long period of time. As inventory is handled in such a harsh condition, two problems are observed in regard to the occupational safety and real-time worker locations, as illustrated in Fig. 1. Firstly, there is a lack of systematic measure to define the health status of workers when they are working in the cold storage facilities. Currently, the health status is measured by using standard operating procedures (SOPs) in a regular basis. However, it is inadequate to determine the health status of workers based on the personal health information, such as heart rates, ages, and weights. It may lead to high risks in product handling and ineffective warehouse management because of poor monitoring and unguarded facility hazards [4]. Secondly, it is lack of real-time positioning of warehouse workers, such that it is difficult to locate the workers if any accidents occur. The prompt rescue attempt will be delayed. This problem becomes more challenging if the scale of warehouse is large.
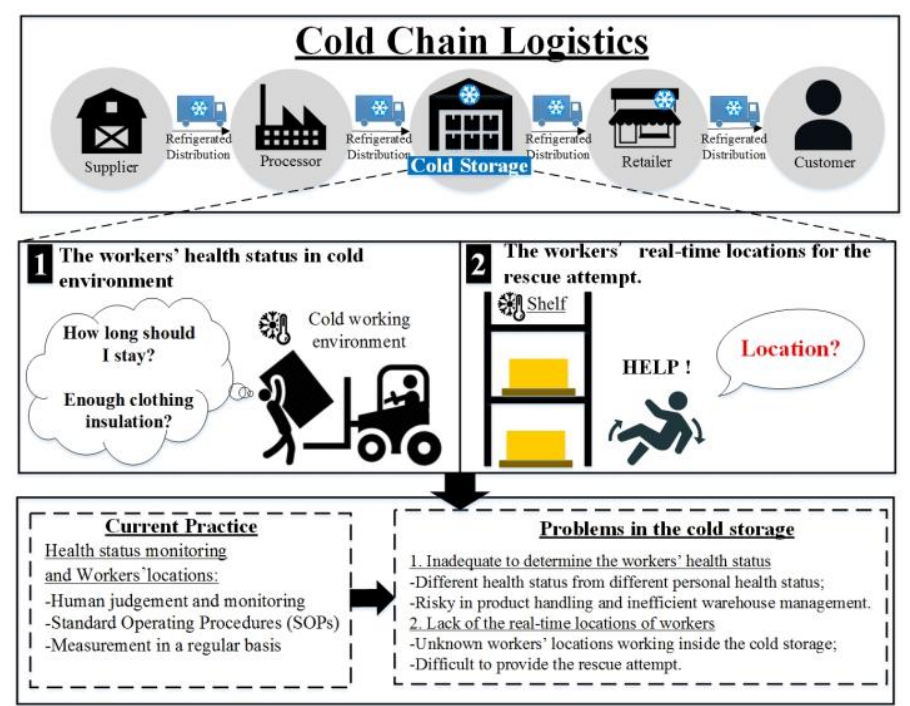

Fig. 1. Occupational problems in cold storage facilities

Currently, ISO11079 is an international standard for determination and interpretation of cold stress in terms of 
duration limited exposure. Duration limited exposure refers to the time limits allowed to work in cold. Although ISO11079 provides a standard to evaluate the cold stress and duration limited exposure when working in a cold environment, the parameters are inputted manually and the outputs are not customized for workers with different health status. It lacks the flexibility to adjust the duration limited exposure with different physiques, and provide responsiveness to the real-time situations. Therefore, there is a need to have automatic data capturing tools, i.e. RFID, integrating to an IoT system for data analytics. In this paper, an IoT-based occupational safety management system (IoT-OSMS) is proposed, with the adoption of Bluetooth Low Energy (BLE) and sensing techniques, to determine the workers' locations and health status. Through the artificial intelligence (AI) technique, i.e. fuzzy logic approach, cold stress and monitoring review cycle can be determined to adjust the estimates of ISO 11079, thus supporting the functionalities of occupational safety management. The duration limited exposure and monitoring review can be customized according to the personal health status of workers. On the other hand, real-time workers' locations help to plan the rescue attempt if necessary. Therefore, the occupational safety can be guaranteed by monitoring their real-time locations and cold stress with a dynamic monitoring review cycle.

This paper is divided into six sections. Section II is the literature review which covers the overview of cold chain, IoT in traceability, and AI techniques for decision support. The system design of IoT-OSMS follows as Section III. Section IV is a case study to investigate the feasibility of the proposed system, followed by the results and discussion of IoT-OSMS in Section V. Conclusions are drawn in Section VI.

\section{LITERATURE REVIEW}

This section reviews the literature related to the design of the IoT-OSMS in cold storage facilities. It consists of (i) overview of cold chain, (ii) IoT in traceability, and (iii) AI techniques for decision support.

\section{A. Overview of cold chain}

To handle the fresh, frozen, and pharmaceutical products, cold chain has played an important role in the parts of storage and transportation [5]. Similar to the traditional supply chain management, the operations in cold chain consist of production, processing, transportation, and distribution. However, cold chain management particularly focuses on information integrity, data transference, and inventory quality [6-7]. Although the freezing $\left(-18^{\circ} \mathrm{C}\right)$ and chilling $\left(0-4^{\circ} \mathrm{C}\right)$ technologies are applied in the storage and transportation, the inventory and environment information has to deliver to all the parties to establish a seamless supply chain integration. When working in a cold environment, the occupational health and safety for warehouse workers is a critical issue in warehouse management. The cold work and environment refer to the situation which works at or below $10^{\circ} \mathrm{C}$ in light physical work, and it may cause some uncomfortable sensations of cold [8]. Tiina and Juhani [9] presented that the health problems in cold workplace are contributed by exposure condition, physical activity, clothing, individual, and socioeconomic information.
It was summarized that the health performance would be decreased, and the probability of morbidity, mortality, and cold injures would be increased. In general practice, ISO 11079 is a measure to determine the cold stress by using the required clothing insulation such that the recommended exposure time can be estimated in the cold workplace [10]. The required data for calculation include metabolic energy production, ambient temperature, air permeability, walking speed, air velocity, humidity, and basic clothing insulation. Although ISO11079 is one of the important measures to evaluate workers' duration limited exposures, the manual data input is time-consuming and containing human errors. It lacks the flexibility and customization to assess the health status of various workers. By integrating automatic data capturing technologies in IoT environment, the data acquisition of the evaluations can be error-free and real-time to generate the results of ISO 11079. To construct an IoT system for information exchange and mining, the wireless communication technologies, such as RFID or BLE, should be used to calculate the cold stress in a real-time manner. Hence, the occupational health and safety can be guaranteed.

\section{B. IoT in traceability}

Internet of Things (IoT) is the state-of-the-art idea to integrate the information of physical materials into the Internet. Pang et al. [11] stated that IoT system is a unique, tailored interconnection system linking to objects with identifying and sensing techniques. Due to the welldevelopment of RFID applications, Wireless Sensor Networks (WSNs), and Smart Things, IoT becomes an active research area with considering the fusion of sensing techniques, efficient wireless communication, and predictive analytics [12]. IoT applications are widely developed in many industries, including the supply chain management. In relation to the traceability in cold storage facilities, IoT/RFID systems have been developed for warehouse operations. Resources tracking and management, such as inventory and forklift trucks, is one of the critical issues in warehouses. It can be accomplished by using the RFID systems [13-14]. Their systems focus on optimizing the resource allocation by developing the decision support systems. In addition, Xiao et al. [15] proposed an improved monitoring system by setting the sensor nodes in cold chain storage and transportation. It is a total solution from the fish catching to market sales with adoption of ZigBee wireless sensor network and compressed sending. Hence, the environmental conditions can be monitored in the whole supply chain, and the shelf life is also predicted to the users. Tsang et al. [16] designed a Bluetooth based indoor positioning system in a simple and rapid approach to locate the people in an indoor environment. However, to the use of IoT to address the problems of occupational health and safety issues in cold chain logistics is limited. Moreover, the predictive analytics can be performed by artificial intelligence (AI) techniques to enhance the prediction accuracy and efficiency. 


\section{AI techniques for decision supports}

Fuzzy logic is one of the AI techniques to perform approximate reasoning processes by converting the vague information into the crisp values, and the results can be expressed in the form of linguistic terms [17]. Domain experts have to decide the membership functions for the input parameters, and the IF-THEN rules in the knowledge repository. The fuzzy sets are capable to handle the data with uncertain and vague natures, which does not have sharp definition of boundaries [18]. It is used to support the classical mathematical models. Chunshien and Lee [19] presented an auto-warehousing crane system with implementing Fuzzy motion control. The crane system became more accurate in 3 axial dimensions by integrating the engineering experience and judgement. On the other hand, fuzzy logic is applied in the manufacturing organizations to perform the leanness assessment [20]. It aims at preventing the manual computation because of time-consuming and error-prone characteristics. Thus, the above studies show that fuzzy logic applications are capable to support the classical mathematical models by integrating fuzzy sets and expertise.

In summary, according to the aforementioned fields of literature, it is found that the awareness of the occupational health and safety policy should be highlighted for working in cold storage facilities. Hence, an IoT-based occupational safety management system is a feasible solution to locate the workers and estimate the cold stress with the integration of fuzzy logic. The cold stress is customized with the age and health information. Therefore, the worker health and safety can be guaranteed, and their performance can be increased.

\section{Design OF THE IOT-BASED OCCUPATIONAL SAFETY MANAGEMENT SYSTEM}

Fig. 2 shows the system architecture of the IoT-based Occupational Safety Management System (IoT-OSMS). It is divided into 4 tiers, namely data acquisition module (DAM), positioning module (PM), fuzzy logic module (FLM), and cold workplace safety program (CWSP).

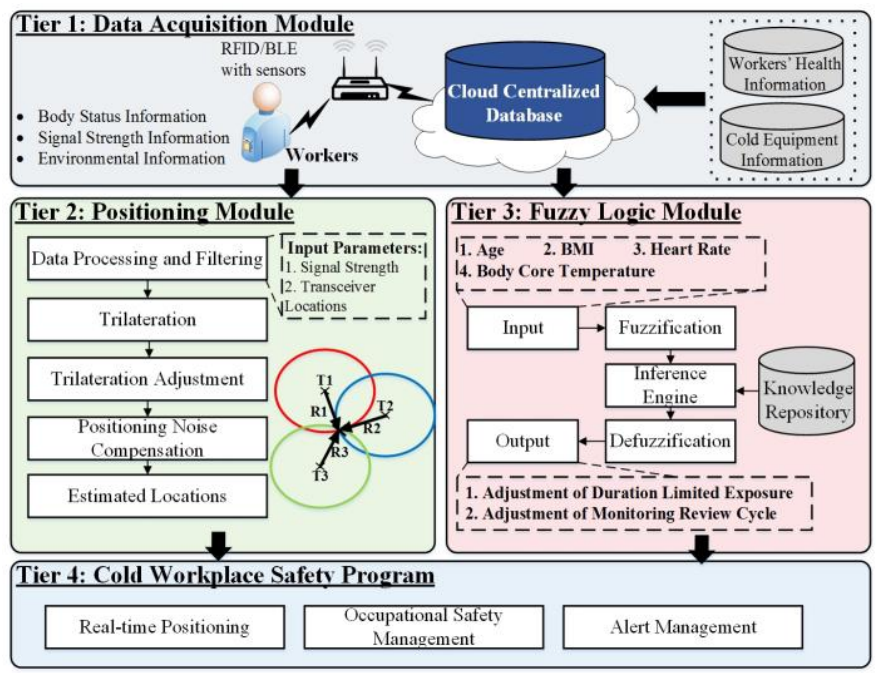

Fig. 2. System architecture of IoT-OSMS

\section{A. Tier 1: Data Acquisition Module (DAM)}

In the data acquisition module, the workers' health and cold equipment data, such as age, height, weight and cold preventive equipment, are firstly collected. These data are classified as static data which do not require real-time update and can be updated in a regular basis. On the other hand, for dynamic data such as positioning of workers, the workers are equipped with a Bluetooth Low Energy (BLE) and sensing wearable hand band. BLE DA14580 is selected to perform the wireless communication in IPv6 over Wireless Personal Area Network (6LoWPAN). Sensing techniques are used to collect the body health status, signal strength, and environmental information, i.e. the ambient and body core temperature, and heart rate. The collected data is stored in a cloud-based centralized database to support the Tire 2 and 3, which is effective to manage the data in a secure manner.

\section{B. Tier 2: Positioning Module (PM)}

In the positioning module, there are five steps to calculate the accurate workers' locations, namely data processing and filtering, trilateration, trilateration adjustment, positioning noise compensation, and estimated locations generation. The characteristics of this positioning module is a simple and rapid approach, which purely uses the received signal strength indicator (RSSI) to compute the 2D locations. Hence, the inputs of this module are signal strength values and transceiver locations. For the data processing and filtering, receiver $(\mathrm{Rx})$ sensitivity and Kalman filtering are applied to eliminate the signal variations for the collected signal strength information. $\mathrm{Rx}$ sensitivity is referred as a minimum acceptable level of signal strength, and it can be defined by following the specification of the electronic modules. The value can be validated by experimenting the RSSI in various distances. The Rx sensitivity of DA14580 is $-93 \mathrm{dBm}$, which means that the collected RSSI values below $-93 \mathrm{dBm}$ are filtered in advance. Secondly, the Kalman filtering technique is used in correcting and predicting the present and future RSSI values. Since the RSSI values are the scalar, the Kalman filter can be simplified to input the initial estimate of $x_{t-1}$ and $P_{t-1}$, referring to the RSSI value and error covariance respectively. As there is no input control in this stage, the scalar Kalman filtering algorithm is expressed in process and measurement as follows.

$$
\begin{gathered}
\mathrm{x}_{t}=A_{t} \mathrm{x}_{t-1}+w_{t} \\
\mathrm{z}_{t}=H_{t} \mathrm{x}_{t}+v_{t}
\end{gathered}
$$

where, in time t, $x$ denotes the RSSI value; $A$ denotes the state transition function; $z$ denotes the measurement in RSSI values; $H$ denotes the sensor function; $w$ and $v$ denote the process and measurement noises respectively. Therefore, the variations of RSSI values can be eliminated.

Theoretically, the corrected RSSI values can be used to calculate the locations by using Trilateration. The RSSI values are converted into the form of distance through the signal propagation model in (3). The locations are estimated by at least three sets of transceivers' RSSI values and coordinates. However, in practice, this method also generates many residuals, and thus the trilateration adjustment is needed to 
reduce the difference between the estimated and true locations. The core concept of trilateration adjustment is that the error terms are expressed by the Taylor's series, and the estimated locations are improved by the distance observation equation, in (4). In the final stage, k-nearest neighbor algorithm is applied to compensate/reduce the positioning noise. It uses the Euclidean distance to define the closest transceivers, and the estimated locations are averaged with the transceivers' coordinates. Hence, the positioning results can be more accurate and close to the true locations.

$$
\begin{gathered}
R S S I(d B m)=A-10 n \times \log (d)+\varepsilon \\
R_{i j}=d \mathrm{~L}_{i j}-\left(L_{i j}-O_{i j}\right)
\end{gathered}
$$

where, in equation $3, A$ is the RSSI value at $1 \mathrm{~m}$ distance; $d$ is the estimated distance, $n$ is path loss exponent; $\varepsilon$ is the error term. In equation $4, L_{i j}$ is the actual distance from point $\mathrm{i}$ to $\mathrm{j}$; $\mathrm{O}_{i j}$ is the observed distance from point $\mathrm{i}$ to $\mathrm{j}$, which is referred to $d ; R_{i j}$ is the residual error; $d L_{i j}$ represents the summation of partial derivative of $L_{i j}$ with respect to $x_{i}, x_{j}, y_{i}$, and $y_{j}$.

\section{Tier 3: Fuzzy Logic Module (FLM)}

In the cold storage facilities, the workers are lack of the information related to exposure duration and sufficiency of the clothing insulation. ISO 11079 proposed that the duration limited exposure can be estimated by nine parameters [10]. However, the model is assumed that the workers are 30 years old, $70 \mathrm{~kg}$ (male)/60 kg (female), and $1.75 \mathrm{~m}$ tall (male) $/ 1.7 \mathrm{~m}$ tall (female). It also does not include the factors of workers' health and environmental conditions. To make the appropriate adjustments, fuzzy logic is applied with the input parameters, including age, Body Mass Index (BMI), heart rates, and body core temperature. The mechanism of fuzzy logic can be divided into three sections, namely fuzzification, inference engine, and defuzzification. In fuzzification, the input parameters are converted into fuzzy sets and defined by the membership functions, such that the numeric data becomes the linguistic terms. The input fuzzy sets are proceeded to the inference engine to generate the output fuzzy sets. The knowledge repository is to support the inference engine with the pre-defined knowledge in the form of IF-THEN rules. In general, the knowledge is collected from the experience or expertise of the cold storage facilities, such as the experienced workers and managers. Afterwards, in defuzzification, the output fuzzy sets can be converted from the linguistic terms to numeric data for further applications. The output parameters of FLM are the adjustments of duration limited exposure and monitoring review cycle. Therefore, the results of FLM can support the cold workplace safety program together with PM.

\section{Tier 4: Cold Workplace Safety Program (CWSP)}

In this module, the outputs from PM and FLM are integrated to become a solution with regard to the occupational health and safety issue. There are three components to guarantee the occupational safety for the workers, namely real-time positioning, occupational safety management, and alert management. From PM and FLM, the workers' locations and health status have been estimated in a real-time manner. Moreover, the duration limited exposure and monitoring review cycle are customized for the workers. The managers can understand the current workers' situations inside the cold storage facilities easily. Hence, the managers can strictly follow the customized duration to provide the appropriate clothing insulation and rests, and to prevent that the workers stay too long in the cold storage. In addition, the alert management provide the warning signals when workers are identified as the risky/dangerous level. Therefore, the workers' occupational health and safety can be improved and guaranteed.

\section{CASE STUdy}

In this section, a case study is conducted in Chevalier Cold Storage and Logistics Limited, which is located in Hong Kong for providing cold chain services. As the employees have wide range of age and BMI, the customized duration limited exposure and monitoring review cycle are important to guarantee their health and safety.

\section{A. Company Background}

Chevalier Cold Storage and Logistics Limited is a third party logistics (3PL) service provider in Hong Kong, specialized in cold supply chain logistics operations. The company was founded in 2002 and boasts an 18-storey, 28,000 metric tons capacity building. The warehouses include freezer, chiller, climate-controlled, bonded, and international standard fine wine sections. In the toughest environment, the freezer's temperature is kept at $-16^{\circ} \mathrm{C}$ to $-20^{\circ} \mathrm{C}$ in order to meet the requirements to store meat, seafood and other kinds of frozen food. In the chiller, the temperature is maintained at $1-4^{\circ} \mathrm{C}$ for storing vegetables, fruit and dairy products. In response to the growing demand of cold chain services, the company was accredited and upgraded to ISO 9001:2008. Thus, the workflow and operation procedures have been standardized to provide a high level of quality service. Therefore, it provides comprehensive services alongside with the provision of transportation, one-stop logistics services, and other professional cold chain services.

\section{B. Problems existing in the company}

At present, the warehouse workers are responsible for manual operations, such as put-away, picking, sorting, and packing in the large-scale cold environment. Without the appropriate clothing insulation, it is hazardous to their performance, health and safety. In practice, each cold storage facility has two warehouse workers to handle the operations, in which one is responsible for storing and retrieving, and the other one is responsible for waiting outside for any accidents and taking the inventory to the docking bay. However, during the peak season, this ideal situation may not happen such that the workers are busy in handling the inventory. Although the company has installed CCTV surveillance at the entrance of the cold storage facilities, it is insufficient to monitor the operations inside the facilities. To summarize, there are lack of real-time worker positioning, customized duration limited exposure, and monitoring review cycle to establish the cold workplace safety plan in the cold storage facilities. 


\section{Implementation of IoT-OSMS}

A pilot study of implementing IoT-OSMS in Chevalier Cold Storage and Logistics Limited is conducted to investigate the performance of real-time positioning, customized duration limited exposure, and monitoring review cycle to guarantee the occupational health and safety for workers. The 5-step implementation roadmap is listed in the Fig. 3.

Implementation Roadmap

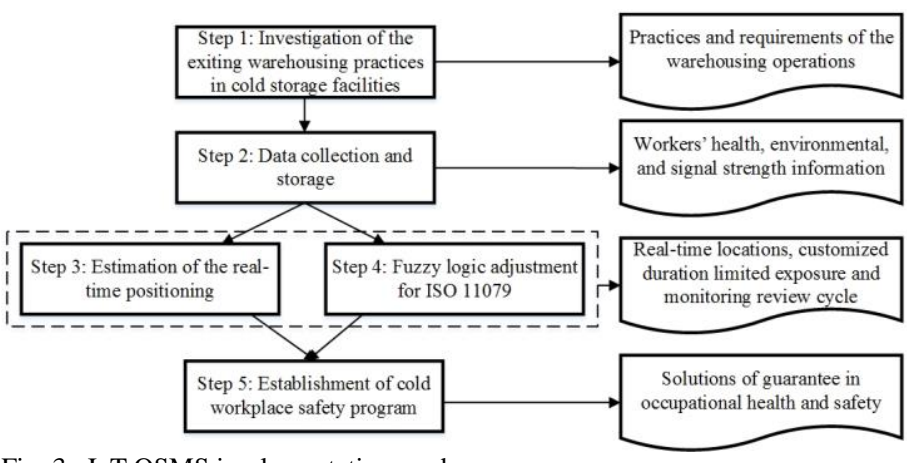

Fig. 3. IoT-OSMS implementation roadmap

In step 1, the exiting warehouse practice is that two workers are assigned in one cold warehouse. They can monitor each other, and decide the rescue attempts if necessary. They are responsible for receiving, put-away, sorting, picking, and packing, which follow the SOPs.

In step 2, the workers are required to wear the BLE handbands to collect the information of worker' health status, signal strength, and environment in the cold storage facilities. The data is then transferred to the cloud database through the network gateway. Basic health and cold equipment information is also collected and stored in the database which supports the computations of PM and FLM to establish the CWSP.

In step 3, the signal propagation model is formed in (5). It is used to convert the RSSI values to the corresponding radii.

Signal

Propagation $\quad$ RSSI $=-11.5 \ln ($ Distance $)-54.331$

Model:

When setting three stationary transceivers in $(0,0),(4,0)$, and $(0,4)$ with 140 collected RSSI values, the location is estimated in MATLAB $^{\circledR}$ environment and compared to the true location of $(2,2)$. The positioning result of $\mathrm{x}^{-}$and $\mathrm{y}$ coordinates is shown in Fig. 4.

In summary, the changes of standard deviation and positioning error in observed and estimated locations are reduced by $85.8 \%$ and $12.6 \%$.

In step 4 , the MATLAB ${ }^{\circledR}$ fuzzy logic toolbox is applied to define the input and output parameters, and the IF-THEN rules in the knowledge repository. The numeric input parameters are converted into the fuzzy input sets with the defined ranges of membership function. Hence, the outputs related to the adjustments of duration limited exposure and monitoring review cycle can be generated. Table I shows the defined parameters and their membership functions for the fuzzy logic toolbox. The values and types of membership functions are defined by the domain expert in cold storage facilities.
Referring to a set of worker's information as an example, the worker is 28 years old, with 27 in BMI, 52 times/min in heart rates, and $33.5^{\circ} \mathrm{C}$ for the body core temperature, by using the designed FLM, it is suggested that the duration limited exposure and monitoring review cycle should be reduced by $50 \%$, as shown in Fig. 5.
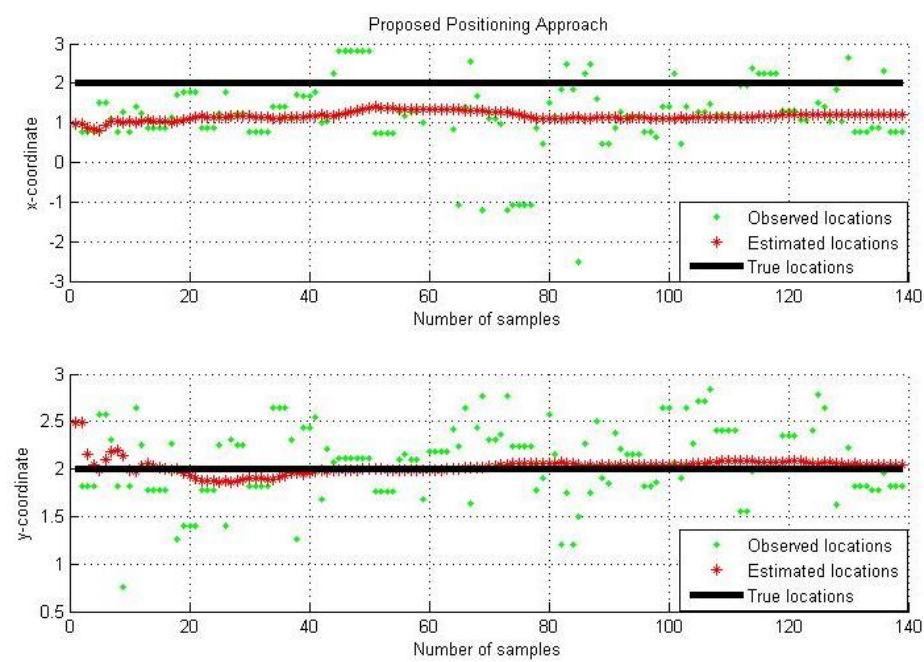

Fig. 4. (2,2) estimated positioning result in PM

In step 5, according to ISO 11079, the duration limited exposure can be estimated in a neutral value. The warehousing operations are being done at environment of $-18^{\circ} \mathrm{C}$ and $60 \%$ relative humidity, with $8 \mathrm{l} / \mathrm{m}^{2} \mathrm{~s}$ of air permeability and $0.4 \mathrm{~m} / \mathrm{s}$ walking speed and relative air velocity. When the workers dress the 3-layer clothing with insulated jacket and trousers, the neutral duration limited exposure is $1.2 \mathrm{hrs}$, and the required recovery time is $0.6 \mathrm{hrs}$. Hence, the duration limited exposure should be adjusted to $0.6 \mathrm{hrs}$. In addition, if the original monitoring review cycle is $1 \mathrm{hr}$, it should be reduced to $0.5 \mathrm{hrs}$. In other words, the maximum duration in cold storage is $0.6 \mathrm{hrs}$, and the monitoring will be conducted again after a half hour. The workers will receive these information, and their real-time positioning can be visualized for supporting the safety program, such as rescue attempt.

TABLE I. The information of parameters for FLM

\begin{tabular}{|c|c|c|c|c|}
\hline \multirow{15}{*}{ Input } & Parameters(Unit)[Symbol] & Fuzzy class & Membership function & Type \\
\hline & \multirow{3}{*}{ Age $[\mathrm{A}]$} & Low & {$[18,25,32]$} & Triangle \\
\hline & & Average & {$[25,32,51,58]$} & Trapezoid \\
\hline & & High & {$[51,58,65]$} & Triangle \\
\hline & \multirow{3}{*}{ BMI [B] } & Low & {$[15,16.5,18.5]$} & Triangle \\
\hline & & Average & {$[16.5,18.5,24,28]$} & Trapezoid \\
\hline & & High & {$[24,28,30]$} & Triangle \\
\hline & & Low & {$[40,40,50,60]$} & Trapezoid \\
\hline & Heart rate & Relative low & {$[50,60,70]$} & Triangle \\
\hline & \multirow[t]{3}{*}{ (unit/min) $[\mathrm{C}]$} & Average & {$[60,70,80]$} & Triangle \\
\hline & & Relative high & {$[70,80,90]$} & Triangle \\
\hline & & High & {$[80,90,100,100]$} & Trapezoid \\
\hline & Body core temperature & Low & {$[30,30,35.5,36.5]$} & Trapezoid \\
\hline & \multirow[t]{2}{*}{$\left({ }^{\circ} \mathrm{C}\right)[\mathrm{D}]$} & Average & {$[35.5,36.5,37.5]$} & Triangle \\
\hline & & High & {$[36.5,37.5,40,40]$} & Trapezoid \\
\hline \multirow{7}{*}{ Output } & & Substantially decrease & {$[-1,-0.75,-0.5]$} & Triangle \\
\hline & Duration Limited & Significantly decrease & {$[-0.75,-0.5,-0.25]$} & Triangle \\
\hline & Exposure $(\%)[\mathrm{E}]$ & Slightly decrease & {$[-0.5,-0.25,0]$} & Triangle \\
\hline & & No change & {$[-0.25,0,0.25]$} & Triangle \\
\hline & Monitoring Review & Slightly increase & {$[0,0.25,0.5]$} & Triangle \\
\hline & Cycle (\%)[F] & Significantly increase & {$[0.25,0.5,0.75]$} & Triangle \\
\hline & & Substantially increase & {$[0.5,0.75,1]$} & Triangle \\
\hline
\end{tabular}




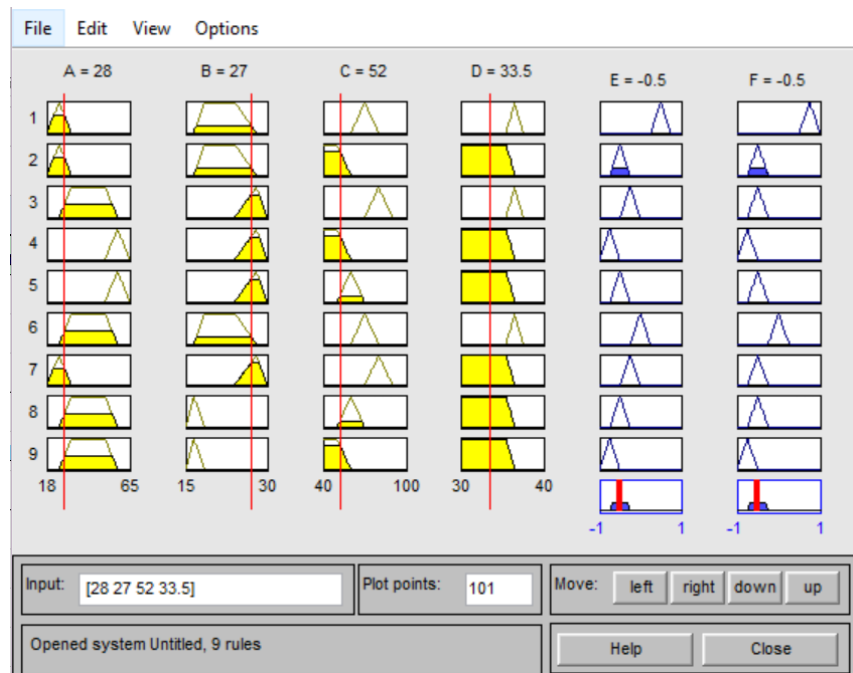

Fig. 5. Example result of FLM

\section{RESULTS AND DISCUSSION}

This paper presents the IoT-OSMS to ensure the occupational health and safety by the adoption of BLE handband to capture the real-time information. The duration limited exposure and monitoring review cycle can be customized according to personal health status of workers, and real-time workers' positioning can be estimated. It is also a method to compensate the model of ISO 11079 by integrating the factors of age, BMI, heart rates, and body core temperature. Through the implementation of IoT-OSMS, it ensures (i) the occupational health and safety, and improves (ii) working performance in cold facilities. Firstly, the workers are monitored under customized duration limited exposure and real-time positioning. Before any accidents/cold injuries occur, the alerts will be sent to the workers when they are at the dangerous situations. In addition, their colleagues can locate and make the appropriate rescue attempt to them if needed. Therefore, the occupational safety can be guaranteed with the help of state-of-art technologies. Secondly, when the duration limited exposure is estimated, it can determine whether the workers' health status is suitable to work in the cold storage. It implies their performance can be maintained in a stable manner. The working performance is not only improved, but also become more stable for work measurement.

\section{CONCLUSIONS}

In order to ensure the occupational health and safety in cold storage facilities, the IoT environment is essential to develop the appropriate occupational safety management with real-time information exchanges and integration. The sensing techniques and wireless communication technologies, i.e. RFID and BLE, are applied to the workers for identifying the position of workers and evaluating the real-time health status. This paper aims at designing an IoT-based occupational safety management system to monitor the workers' health status and locations. Real-time positioning and fuzzy logic are integrated to establish the cold workplace safety program. Fuzzy logic is applied to adjust and customize the duration limited exposure and monitoring review cycle. Hence, the workers' safety can be ensured with appropriate alert and visualization of real-time positioning. In addition, the productivity and working performance can be further improved through implementing this system. As a result, the active monitoring of workers' health and safety can be implemented. The preventive measures can also be done for workers to reduce the probability of getting cold injuries. Therefore, the effort of manual workers' monitoring can be greatly reduced, and the occupational health and safety is further improved and guaranteed.

\section{ACKNOWLEDGMENT}

The authors would like to thank the Research Office of the Hong Kong Polytechnic University and AOC Limited for supporting the project. (Project Code: RUDV)

\section{REFERENCES}

[1] C. Chen, T. Chen, C. Zhang and G. Xie, 'Research on Agricultural Products Cold-Chain Logistics of Mobile Services Application', Computer and Computing Technologies in Agriculture VII, pp. 247-254, 2014.

[2] R. Gormley, M. Brennan and F. Butler, Upgrading the cold chain for consumer food products. National Food Centre, 2000.

[3] K. Likar and M. Jevšnik, "Cold chain maintaining in food trade", Food Control, vol. 17, no. 2, pp. 108-113, 2006.

[4] M.S. Vela-Acosta, P. Bigelow and R. Buchan, "Assessment of occupational health and safety risks of farmworkers in Colorado", Am. J. Ind. Med., vol. 42, no. 2, pp. 19-27, 2002.

[5] The National Development and Reform Commission, 'China Cold Chain Logistics Industry Report, 2014-2017', Research In China, China, 2015.

[6] C. Chen, T. Chen, C. Zhang and G. Xie, "Research on Agricultural Products Cold-Chain Logistics of Mobile Services Application", Computer and Computing Technologies in Agriculture VII, pp. 247-254, 2014.

[7] H. Chen, Y. Li, Y. Mu, L. Wang and X. Zhang, "A Quality Research Analysis of Logistics Distribution Process of Fresh Meat Cold Chain in Beijing", LISS 2013, pp. 697-703, 2013.

[8] ISO15743 (2008) Ergonomics of the thermal environment-Ergonomics of the thermal environment - Cold workplaces - Risk assessment and management. International Organization for Standardization, Geneva.

[9] T.M. MÄKINEN and J. HASSI, "Health Problems in Cold Work", Industrial Health, vol. 47, no. 3, pp. 207-220, 2009.

[10] I. HOLMÉR, "Evaluation of Cold Workplaces: An Overview of Standards for Assessment of Cold Stress", Industrial Health, vol. 47, no. 3, pp. 228-234, 2009.

[11] Z. Pang, L. Zheng, J. Tian, S. Kao-Walter, E. Dubrova and Q. Chen, "Design of a terminal solution for integration of in-home health care devices and services towards the Internet-of-Things", Enterprise Information Systems, vol. 9, no. 1, pp. 86-116, 2013.

[12] S. Li, L. Xu and S. Zhao, "The internet of things: a survey", Information Systems Frontiers, vol. 17, no. 2, pp. 243-259, 2014.

[13] H.K. Chow, K.L. Choy, W.B. Lee and K.C. Lau, "Design of a RFID case-based resource management system for warehouse operations", Expert Systems with Applications, vol. 30, no. 4, pp. 561-576, 2006.

[14] T.C. Poon, K.L. Choy, H.K. Chow, H.C. Lau, F.T. Chanand K.C. Ho, "A RFID case-based logistics resource management system for managing order-picking operations in warehouses", Expert Systems with Applications, vol. 36, no. 4, pp.8277-8301, 2009.

[15] X. Xiao, Q. He, Z. Fu, M. Xu and X. Zhang, "Applying CS and WSN methods for improving efficiency of frozen and chilled aquatic products monitoring system in cold chain logistics", Food Control, vol. 60, pp. 656-666, 2016. 
[16] P.Y.P. Tsang, C.H. Wu, W.H. Ip and Y.K. Tse, "A Bluetooth-based Indoor Positioning System: A Simple and Rapid Approach", Annual Journal IIE(HK), vol. 35, no. 2014/2015, pp.11-26, 2015.

[17] M.A. Nofal and K.M. Fouad, "Developing Web-based Semantic and Fuzzy Expert Systems using Proposed Tool", International Journal of Computer Applications, vol. 112, no. 7, pp. 38-45, 2015.

[18] H. Nguyen and E. Walker, A first course in fuzzy logic. Boca Raton: CRC Press, 1997.
[19] C. Li and C.Y. Lee, "Fuzzy motion control of an auto-warehousing crane system", IEEE Trans. Ind. Electron., vol. 48, no. 5, pp. 983-994, 2001.

[20] S. Vinodh and S.R. Balaji, "Fuzzy logic based leanness assessment and its decision support system", International Journal of Production Research, vol. 49, no. 13, pp. 4027-4041, 2011. 